OPEN ACCESS

Edited by:

Felipe Fregni,

Harvard Medical School,

United States

Reviewed by:

Prasad Shirvalkar,

University of California San Francisco,

United States

Danielle D. DeSouza,

Stanford University, United States

*Correspondence: Alexandre F. DaSilva adasilva@umich.edu

Specialty section This article was submitted to

Neuropathic Pain,

a section of the journal

Frontiers in Pain Research

Received: 19 October 2021

Accepted: 13 January 2022

Published: 07 February 2022

Citation:

DaSilva AF, Datta A, Swami J, Kim DJ, Patil PG and Bikson M (2022) The

Concept, Development, and

Application of a Home-Based

High-Definition tDCS for Bilateral Motor Cortex Modulation in Migraine and Pain. Front. Pain Res. 3:798056.

doi: 10.3389/fpain.2022.798056

\section{The Concept, Development, and Application of a Home-Based High-Definition tDCS for Bilateral Motor Cortex Modulation in Migraine and Pain}

\author{
Alexandre F. DaSilva ${ }^{1 *}$, Abhishek Datta ${ }^{2}$, Jaiti Swami ${ }^{3}$, Dajung J. Kim ${ }^{1}$, Parag G. Patil ${ }^{4,5,6,7}$ \\ and Marom Bikson ${ }^{3}$ \\ ${ }^{1}$ Headache and Orofacial Pain Effort Lab, Department of Biologic and Materials Sciences and Prosthodontics, University of \\ Michigan School of Dentistry, Ann Arbor, MI, United States, ${ }^{2}$ Soterix Medical Inc., New York, NY, United States, ${ }^{3}$ Neural \\ Engineering Laboratory, Department of Biomedical Engineering, The City College of New York, New York, NY, United States, \\ ${ }^{4}$ Department of Neurosurgery, University of Michigan, Ann Arbor, MI, United States, ${ }^{5}$ Department of Neurology, University of \\ Michigan, Ann Arbor, MI, United States, ${ }^{6}$ Department of Biomedical Engineering, University of Michigan, Ann Arbor, MI, \\ United States, ${ }^{7}$ Department of Anesthesiology, University of Michigan, Ann Arbor, MI, United States
}

Whereas, many debilitating chronic pain disorders are dominantly bilateral (e.g., fibromyalgia, chronic migraine), non-invasive and invasive cortical neuromodulation therapies predominantly apply unilateral stimulation. The development of excitatory stimulation targeting bilateral primary motor (M1) cortices could potentially expand its therapeutic effect to more global pain relief. However, this is hampered by increased procedural and technical complexity. For example, repetitive transcranial magnetic stimulation (rTMS) and $4 \times 1 / 2 \times 2$ high-definition transcranial direct current stimulation $(4 \times 1 / 2 \times 2 \mathrm{HD}$-tDCS $)$ are largely center-based, with unilateral-target focus-bilateral excitation would require two rTMS $/ 4 \times 1 \mathrm{HD}$-tDCS systems. We developed a system that allows for focal, non-invasive, self-applied, and simultaneous bilateral excitatory M1 stimulation, supporting long-term home-based treatment with a well-tolerated wearable battery-powered device. Here, we overviewed the most employed M1 neuromodulation methods, from invasive techniques to non-invasive TMS and tDCS. The evaluation extended from non-invasive diffuse asymmetric bilateral (M1-supraorbital [SO] tDCS), non-invasive and invasive unilateral focal (4×1/2×2 HD-tDCS, rTMS, MCS), to non-invasive and invasive bilateral bipolar (M1-M1 tDCS, MCS), before outlining our proposal for a neuromodulatory system with unique features. Computational models were applied to compare brain current flow for current laboratory-based unilateral $\mathrm{M}^{1}{ }^{1}$ and bilateral $\mathrm{M}^{2}{ }^{2} \mathrm{HD}$-tDCS models with a functional home-based $\mathrm{M} 1^{1-2} \mathrm{HD}$-tDCS prototype. We concluded the study by discussing the promising concept of bilateral excitatory M1 stimulation for more global pain relief, which is also non-invasive, focal, and home-based.

Keywords: tDCS, high-definition, chronic pain, home-based, bilateral stimulation, M1 stimulation, migraine 


\section{INTRODUCTION}

Pain perception and sensitivity are generally considered adaptive for our survival. However, whatever the sources, some pain conditions become chronic, even with no clear cause (e.g., tissue damage, infection, or inflammation) (1). Chronic pain does not necessarily restrict to one particular location or origin. Instead, chronic pain commonly expands diffusely throughout the body (2) translating into severe levels of disability and suffering for individuals (3). In this review, we focus mainly on chronic primary pain, which includes chronic widespread pain (fibromyalgia), chronic primary headaches, or orofacial pain disorders (4). Their often bilateral manifestation may stem from central sensitization related to augmented central processing, decreased inhibition of painful stimuli or both despite no clear link to inflammation or nerve damage (5). One of the most common and impactful pain disorders is migraine, which can progress to more than 15 headache days per month referred to as chronic migraine. Although headache localized to one side is a key clinical feature of migraine, at least one-third of patients have bilateral headache (6), and it occurs more frequently in patients with near-daily attacks ( 25 or more days/month) compared to other chronic migraineurs (7).

When widespread pain and headaches experiences become more severe and frequent, they tend to also be associated with multiple comorbidities that are detrimental to the patients' quality of life (e.g., emotional and social dysfunction). This worsens the risk for polypharmacy and medication overuse that iatrogenically can lead to serious adverse effects like opioid addiction $(8,9)$. Hence, non-pharmacological pain therapies may not only be a safer option but also, most importantly, a practical pathway to scale back medication misuse in highly impacted pain patients. Neuroimaging research has elucidated particular brain regions and systems directly or indirectly associated with pain processing and analgesia. The primary motor cortex (M1) has been frequently investigated as a potential cortical target for pain relief (10). When analyzing pain neuroimaging data, researchers commonly flip the brains of the patients to match the cortical sides contralateral to the worst pain, independent of the ipsilateral response. Hence, many studies have over-emphasized the role of the most affected cortical sides. While neuromodulatory M1 protocols have been refined over decades with encouraging clinical outcomes (11), ongoing research is directed toward developing more precise technologies and enhancing consistency. We noticed two main factors limiting effectiveness and reliability in M1 pain neuromodulation when translating directly from the laboratory to the clinical setting, (1) the unilateral delivery of M1 stimulation invasively and non-invasively; and (2) for those approaches requiring hospital and laboratory-based application, the total number of sessions that can be practically delivered non-invasively.

A range of neuromodulation technologies have been established or investigated for pain management. We focus here on those that apply electrical stimulation to M1. Invasive approaches use implanted electrodes, while non-invasive approaches apply stimulation transcranially.
Non-invasive approaches include transcranial magnetic stimulation (TMS) and transcranial electrical stimulation (tES). The most common form of tES trialed for pain is transcranial Direct Current Stimulation (tDCS). tES/tDCS can be delivered using pad (sponge) electrodes (12), with the intervention typically described by the positioning of the two electrodes [e.g., M1-supra-orbital (SO)]. tES/tDCS can alternatively be provided through smaller gel-based "High-Definition" (HD) electrodes $(13,14)$, with the intervention typically described by the configuration of the arrays of $\mathrm{HD}$ electrodes [e.g., $4 \times 1$ $(15,16), 2 \times 2(17)]$. In this review, we follow standard tES nomenclature (18), including "lateralized" for approaches where hemispheres receive symmetric but opposite polarity stimulation but adopt "diffuse asymmetric bilateral" where only one motor region is targeted. Non-invasive "unilateral focal" (sparring the opposite hemisphere) stimulation can be achieved with specific HD or TMS approaches. "Bilateral excitatory" stimulation thus refers to a particular case where motor regions in both hemispheres are excited (e.g., with same waveform/polarity) with a symmetric montage.

Current M1 neuromodulation techniques primarily target the contralateral side of dominant pain, which is rational when addressing focused neuroscientific-driven hypotheses (e.g., what waveform produces short-term changes in experimental motor excitability). Applying neuromodulation protocols developed based on experimental unilateral modulation of M1 excitability to pain management is a rational starting point, but arguably is not optimized to broad pain indications. Specifically, this raises the question of relying solely on unilateral stimulation for deriving critical changes required for satisfactory clinical outcomes and reverting or ameliorating concurrent hyper-excitability, especially in patients with having bilateral or widespread nociplastic pain as in chronic migraine or fibromyalgia (5). Moreover, it prompts us to hypothesize whether we could see differential clinical outcomes by comparing bilateral and unilateral M1 stimulation protocols by pain side/location, and global pain.

Here, we overview the most employed M1 neuromodulation methods, including TMS and tDCS. Then, we suggest strategies to improve the clinical efficacy and feasibility for pain management by introducing (1) scientific rationale/computational modeling of tDCS with enhanced focality over "bilateral" excitatory M1 ${ }^{2} \mathrm{HD}$-tDCS; and (2) homebased focused $M 1^{2} \mathrm{tDCS}$, which enables an increasing number of session for extended treatment and replaces laboratory or clinic settings.

\section{Neuromodulation Approaches for Pain Modulation}

We briefly summarize common neuromodulation techniques applied to acute or chronic pain by categorizing the stimulation methods into unilateral, bilateral bipolar, and bilateral excitatory stimulation (Table 1). This categorization here considers: (a) conventional tDCS approaches using diffuse asymmetric bilateral montages (e.g., M1-SO), (b) unilateral focal stimulation with invasive or non-invasive techniques (e.g., TMS); and 
TABLE 1 | Summary of brain stimulation techniques for pain improvement targeting the motor strip.

\begin{tabular}{|c|c|c|c|c|c|c|c|c|c|c|}
\hline \multicolumn{3}{|c|}{ Techniques } & \multirow{2}{*}{$\begin{array}{l}\text { Pain type (example references) } \\
\text { - Thalamic pain (19) }\end{array}$} & \multirow{3}{*}{\begin{tabular}{|l|}
$\begin{array}{l}\text { Electrodes/ } \\
\text { modality }\end{array}$ \\
$\begin{array}{l}\text { Permanent four array } \\
\text { electrode }\end{array}$
\end{tabular}} & \multirow{3}{*}{\begin{tabular}{|l|} 
Cortical target \\
Left motor cortex; \\
Contralateral to pain \\
Bilateral motor cortex
\end{tabular}} & \multirow{3}{*}{\begin{tabular}{|l}
$\begin{array}{l}\text { Feasibility } \\
\text { at home }\end{array}$ \\
N \\
N \\
\end{tabular}} & \multirow{3}{*}{\begin{tabular}{|l}
$\begin{array}{l}\text { Non- } \\
\text { surgical }\end{array}$ \\
N \\
N
\end{tabular}} & \multirow{3}{*}{\begin{tabular}{|l|l}
$\begin{array}{l}\text { Non- } \\
\text { significant } \\
\text { risk }\end{array}$ \\
$\mathrm{N}$ \\
$\mathrm{N}$ \\
\end{tabular}} & \multirow{3}{*}{\begin{tabular}{|l}
$\begin{array}{l}\text { Bilateral } \\
\text { bipolar } \\
\text { M1 }\end{array}$ \\
$\mathrm{N}$ \\
$\mathrm{N}$ \\
\end{tabular}} & \multirow{3}{*}{\begin{tabular}{|l|l}
$\begin{array}{l}\text { Bilateral } \\
\text { excitatory } \\
\text { M1 }\end{array}$ \\
N \\
Y \\
\end{tabular}} \\
\hline \multirow[t]{2}{*}{ Invasive } & \multirow[t]{2}{*}{ MCS } & Bipolar iMC & & & & & & & & \\
\hline & & Bilateral symmetric & $\begin{array}{l}\text { - Deafferentation pain (20) } \\
\text { - Dysesthetic pain (21) } \\
\text { - Deafferentation pain (22) }\end{array}$ & & & & & & & \\
\hline \multirow[t]{7}{*}{$\begin{array}{l}\text { Non- } \\
\text { Invasive }\end{array}$} & \multirow[t]{3}{*}{ rTMS } & $\begin{array}{l}\text { High-frequency } \\
\text { rTMS }\end{array}$ & $\begin{array}{l}\text { - Migraine (23-26) } \\
\text { - Chronic facial pain (27) } \\
\text { - Mild traumatic brain injury related headaches (28) } \\
\text { - Central Neuropathic Pain (29) } \\
\text { - Hemichorea-pain in left shoulder (30) } \\
\text { - Central pain in spinal cord injury (31) } \\
\text { - Fibromyalgia (32, 33) } \\
\text { - Central and phantom limb pain (34) } \\
\text { - Chronic neurogenic pain (35) }\end{array}$ & Figure-of-eight coil & $\begin{array}{l}\text { Left motor } \\
\text { cortex; Right motor } \\
\text { cortex; Motor cortex } \\
\text { contralateral to pain }\end{array}$ & N & Y & N & N & $N$ \\
\hline & & $\begin{array}{l}\text { Low-frequency } \\
\text { rTMS }\end{array}$ & $\begin{array}{l}\text { - Phantom limb pain (36) } \\
\text { - Chronic neuropathic pain (37) } \\
\text { - Central pain }(38,39) \\
\text { - Deafferentation pain (40) }\end{array}$ & Figure-of- eight coil & $\begin{array}{l}\text { Left motor cortex; Motor } \\
\text { cortex contralateral to pain }\end{array}$ & N & Y & N & N & $N$ \\
\hline & & Deep rTMS & $\begin{array}{l}\text { - Diabetic neuropathy (41) } \\
\text { - Neuropathic pain (42) }\end{array}$ & Hesed (H)-coil & $\begin{array}{l}\text { Lower limb region of the } \\
\text { motor cortex }\end{array}$ & N & Y & N & Y & $N$ \\
\hline & \multirow[t]{4}{*}{ tDCS } & $\begin{array}{l}\text { Conventional } 1 \times 1 \\
\text { tDCS }\end{array}$ & $\begin{array}{l}\text { - Central Pain in traumatic spinal cord injury (43) } \\
\text { - Knee osteoarthritis (44) } \\
\text { - Fibromyalgia }(45) \\
\text { - Migraine }(46,47) \\
\text { - Post-stroke chronic limb pain (48) }\end{array}$ & $\begin{array}{l}\text { Direct current } \\
\text { electrodes with } \\
\text { saline-soaked sponges }\end{array}$ & $\begin{array}{l}\text { Left motor cortex (M1-SO } \\
\text { montage); } \\
\text { Left and right motor } \\
\text { cortex for bilateral bipolar }\end{array}$ & Y & Y & Y & Y & $N$ \\
\hline & & $4 \times 1 \mathrm{HD}-\mathrm{tDCS}$ & - Fibromyalgia $(49,50)$ & \multirow{2}{*}{$\begin{array}{l}\mathrm{Ag} / \mathrm{AgCl} \text { sintered ring } \\
\text { electrodes }\end{array}$} & Left motor cortex & N & Y & Y & N & $N$ \\
\hline & & $2 \times 2$ HD-tDCS & - Chronic myofascial TMD pain (17) & & Right motor cortex & N & Y & Y & N & $N$ \\
\hline & & $\begin{array}{l}2 \times 2 \text { Bilateral M1 } \\
\text { HD-tDCS }^{*}\end{array}$ & $\begin{array}{l}\text { - Migraine } \\
\text { - Widespread pain such as fibromyalgia }\end{array}$ & $\begin{array}{l}4 \mathrm{~cm} \times 1 \mathrm{~cm} \text { strip } \\
\text { electrodes }\end{array}$ & Left and right motor cortex & Y & Y & Y & Y & Y \\
\hline
\end{tabular}

HD, high-definition; IMC, ipsilateral motor cortex; MCS, motor cortex stimulation; M1-SO, primary motor cortex and contralateral supraorbital area; rTMS, repetitive transcranial magnetic stimulation; tDCS, transcranial direct current stimulation.

${ }^{*}$ Proposed home-based non-invasive HD-tDCS montage for bilateral M1 stimulation. 
(c) approaches producing bilateral symmetric excitation. We confine our review to studies utilizing non-invasive transcranial techniques such as tDCS and TMS, and invasive intracranial techniques such as motor cortex stimulation (MCS).

\section{Diffuse Asymmetric Bilateral}

Transcranial direct current stimulation (tDCS) delivers a few milliampere $(\mathrm{mA})$ of electrical stimulation across the scalp to modulate brain excitability, with the predominance of neurophysiological evidence in fact for M1 neuromodulation (51). A single session produces lasting changes that can be prolonged with repetitive (daily) tDCS applications (52). tDCS requires two electrodes (one anode and one cathode) with terminology referring only to the presumed electrode of interest (18), e.g., anodal tDCS of M1. Nonetheless, the "return" electrode remains active, and its placement (typically on the contralateral hemisphere) is presumed to create lateralized stimulation. Generally, and for our purposes, unilateral M1 tDCS refers to the placement of the "return" electrode not on the contralateral motor region, but rather typically over the contralateral supraorbital region. M1 tDCS has been reported to provide pain relief, especially to migraine [see the reviews of $(53,54)]$, temporomandibular disorders $(17)$, and cancer patients (55-57), and has even shown immediate activation of the opioid system like MCS (58).

Accumulating evidence suggests the diffuse asymmetric bilateral tDCS targeting M1 is a promising tool for modulating pain, including after-surgery pain and chronic pain disorders such as fibromyalgia, neuropathic pain, phantom pain, and migraine. For instance, tDCS intervention significantly decreased patient-controlled opioid usage compared to sham after total knee arthroplasty (59) or lumber surgery (60). In fibromyalgia patients, M1 tDCS yielded more beneficial effects than dorsolateral prefrontal cortex (DLPFC) tDCS in terms of pain relief or quality of life (45) and a longer-lasting clinical efficacy of up to 1-2 months after the tDCS (61). tDCS can also potentially be used in chronic migraine showing efficacy as a preventive treatment (46). In that study, there was a significant reduction in pain intensity and length of migraine episodes after ten sessions of tDCS treatment. Moreover, pain levels continued to decrease as far as 120 days after the end of treatment.

While diffuse asymmetric bilateral M1 tDCS has a great potential in modulating pain, heterogeneity across the studies partly due to a lack of standardized protocols, and in some cases limited sample sizes, short-term treatment, and follow-up days (62), should be addressed as part of ongoing optimization and validation efforts.

\section{Unilateral Focal M1 Stimulation-Invasive (MCS) and Non-invasive (TMS and HD-TDCS)}

Invasive MCS has been investigated since the early 1990s as a treatment of last resort for patients with refractory pain (19). Despite some side effects (e.g., infection or hardware dysfunction) and controversies regarding efficacy (63), the surgical placement of epidural electrodes in the M1 has provided pain relief for patients with chronic neuropathic pain of various different locations and origins $(64,65)$, typically unilateral. For this reason and because it is highly invasive, MCS is usually restricted to the modulation of M1 contralateral to the pain side, or worse side when bilateral. In fact, scientific reports of bilateral MCS are scarce. When non-invasive technologies were developed, they tentatively mirrored MCS as a guide for their protocols, and thus adopted largely unilateral approaches. Noninvasive approaches similarly adopted unilateral stimulation for reasons of technical or protocol expediency. A further reason for non-invasive approaches employed unilateral stimulation is that they were followed by classical experimental protocols to modulate M1 with scientific rigor (66). It is thus important to note that approaches using unilateral M1 stimulation were not explicitly rationalized for the clinic under the assumption that just unilateral stimulation would be superior in bilateral pain disorders.

TMS in a non-invasive technology initially developed specifically to allow tolerated supra-threshold stimulation of the motor cortex (67), with repetitive protocols (rTMS) producing increased motor cortex plasticity of relevance to treatments (6870). The ability to direct TMS for targeted cortex stimulation has been a long-standing focus, such as the development of figure-of-eight coils (71). Thus, the application of rTMS for pain treatment through M1 stimulation almost exclusively adopted focal approaches that were inherently unilateral.

Compared to conventional tDCS with the anode over M1 and the cathode over the contralateral supraorbital area, highdefinition (HD)-tDCS has been more recently developed to increase focality at the target area. With HD electrodes arranged in arrays, researchers have greater flexibility to modulate excitability than in conventional tDCS (72). This unilateral focal M1 tDCS can precisely target the homuncular M1 face and head area (17, 73), hand area (74), and lower limbs (75) based on pathological brain state and study purposes. In this way, researchers can optimize the effect with flexible use of HD electrodes (smaller than conventional, $<5 \mathrm{~cm}^{2}$ ), which overcomes the low spatial resolution of conventional tDCS (14, $16,73,76)$. In addition, HD-tDCS was more efficient in inducing longer-lasting neuroplasticity than conventional tDCS (16). A common HD montage is $4 \times 1 \mathrm{HD}$-tDCS with one electrode surrounded by four electrodes with opposite polarity, producing focal unilateral cortical stimulation $(15,16,77)$. The clinical application of $4 \times 1 \mathrm{HD}$-tDCS to fibromyalgia patients has shown to reduce pain intensity in different studies $(49,50)$. Approaches using just $2 \mathrm{HD}$ electrodes for specific unilateral M1 modulation have also been developed $(78,79)$ for behavior and cortical excitability examination.

HD-tDCS with a $2 \times 2$ montage ( 2 anodes and 2 cathodes) over the unilateral M1 has been tested on a selected cohort of patients with chronic TMD patients during 5-daily sessions (17, 80). The M1 chosen was contralateral to the worst TMD pain side. There were differences in sensorimotor measurements between the active and sham groups, including the pain visual analog scale (VAS). Compared with the placebo group, the active group had more responders in general pain relief $(>50 \%$ in the VAS) at a 1-month follow-up. Most importantly, there was improvement of the contralateral sensory-discriminative pain measures [e.g., pain intensity, area, and their summation (PAINS)] during the 
treatment week, but not ipsilateral pain measures, suggesting a unilateral and focused M1 HD-tDCS stimulation selectively improved contralateral sensorimotor function.

Despite encouraging (ongoing) trails of neuromodulation targeting M1 pain, outcomes are mixed $(62,81-83)$. We believe optimization of dose to be key, which includes both guiding current to the brain based on rational strategies and developing protocols that allow extensive sessions-the headgear developed here supports these aims. However, the clinical effectiveness of a given dose (compared to others) can only be validated through clinical trials. They are encouraging computational modeling (76), clinical neurophysiology $(78,79)$, and early clinical trials (46) supporting the potency of our principled approach.

\section{Bilateral M1 Excitation}

In a case study of invasive bilateral stimulation over the M1 in a single patient with central dysesthetic pain and intentional tremor, most pain-evoked phenomena and improved steady burning pain and tremor were eliminated 2 month after permanent placement of MCS (21). Another single case study showed pain relief with bilateral MCS applied for deafferentation pain following spinal cord injury, suggesting that bilateral MCS could be a potentially useful treatment option for deafferentation pain (22).

There are few studies that use non-invasive stimulation over bilateral M1 for pain relief in chronic pain. Onesti et al. (41) studied the effect of 5 days of rTMS on pain relief in 23 diabetic neuropathic patients with Hesed coil (H-coil), which stimulates deeper cortical area bilaterally (41). They showed that active rTMS produced more significant pain reduction which lasted 3 weeks than sham stimulation. In a later study conducted by Shimizu et al. (42), they examined the efficacy of five daily sessions of rTMS with H-coils for lower limbc neuropathic pain, showing pain reduction 1-hour after rTMS stimulation (42). This analgesic effect did not last for more than 2 weeks, suggesting a need for revised protocols (e.g., increased number of sessions) to deliver long-term benefit to patients.

Bilateral HD-tDCS stimulation may be an effective approach for chronic pain based on recent work in healthy participants. For instance, bilateral anodal tDCS over the tongue M1 in healthy individuals induced more enhanced cortical excitability and tongue motor function than unilateral stimulation (84). Also, in a later study with healthy individuals, multifocal tDCS with bilateral M1 as anodes reverted an inhibited corticomotor excitability and impaired conditioned pain modulation that had been induced by topical experimental pain (85).

It is known that $\mathrm{tDCS}$ modulates resting membrane potential excitability, which outlasts a few hours or more, inducing neuroplasticity as evidenced by neurochemical or blood-oxygenlevel-dependent (BOLD) signal changes in a number of cortical or subcortical regions $(58,86-91)$. Patients with chronic pain exhibit altered brain excitability which contributes to central sensitization features (e.g., augmented pain processing or deficient inhibitory pain modulation) (92). Neuroimaging evidence suggested that M1 tDCS with conventional montages modified $\mu$-opioid receptor availability (58), Glx (glutamate and glutamine) level (87), or BOLD signal responses (90) of the descending pain modulatory pathway, including periaqueductal gray and anterior cingulate cortex, associated with pain sensitivity or secondary hyperalgesia. We observed that HD-M1 tDCS, in particular, reduced the contralateral side of TMD pain (17). We speculated that this analgesic effect would be delivered through the indirect/direct modulation of the ipsilateral side of the brain with highly-focused stimulation $(16,73)$.

Considering that precisely targeted $\mathrm{tDCS}$ over unilateral M1 for pain worked to partly echo how invasive MCS modulates central pain (locked into unilateral M1), it would be worth examining the effect of bilateral HD-tDCS effect on chronic pain to see if it maximizes the effect of unilateral stimulation.

\section{VALUE OF HOME-BASED HD-TDCS}

Multiple daily visits to a treatment center to participate in a study is not only burdensome for patients, but can pose a critical barrier, especially for those with disabilities or limited access to study sites. Chronic pain requires longterm management; thus, accessibility, which helps patients adhere to the treatment protocol, is critical for achieving long-term changes in neural excitability and connection. In addition, tDCS effects have been shown to be optimized with new montages, more frequent number of session or longer treatment duration, as in studies lasting over 4 weeks $(17,50,54,61,93-95)$. If these therapeutic approaches are implemented properly at home, these efforts could significantly increase and accelerate the clinical effect reported in tDCS trials.

To date, only a few studies have shown the clinical efficacy and feasibility of home-based tDCS in certain conditions (e.g., auditory hallucination, tinnitus, and multiple sclerosis) with devices and instruction for self-administration while remotely monitoring the use of tDCS (96-98). In addition, while a few studies were conducted for pain management, recent homebased neurostimulation for the treatment of fibromyalgia has shown its feasibility and effectiveness in extending the treatment period (up to 60 sessions) and level of pain reduction (99). In another home-based study using real-time monitoring in older people with knee osteoarthritis, the patients benefited from the 10 home-based tDCS sessions through real-time monitoring in terms of reduction in pain severity and sleep disturbances (100).

There is accumulating evidence of rTMS efficacy for managing post-operative pain and chronic pain has shown promise; however, from a practical standpoint, tDCS has specific advantages over TMS, in particular the tolerance to head movement, noise, complications (e.g., risk of seizure), and price. More importantly, deployability in settings like the primary care facility, hospice (101), or home (102), especially amid a pandemic, provides a critical accessibility advantage for those already burdened by disease. 


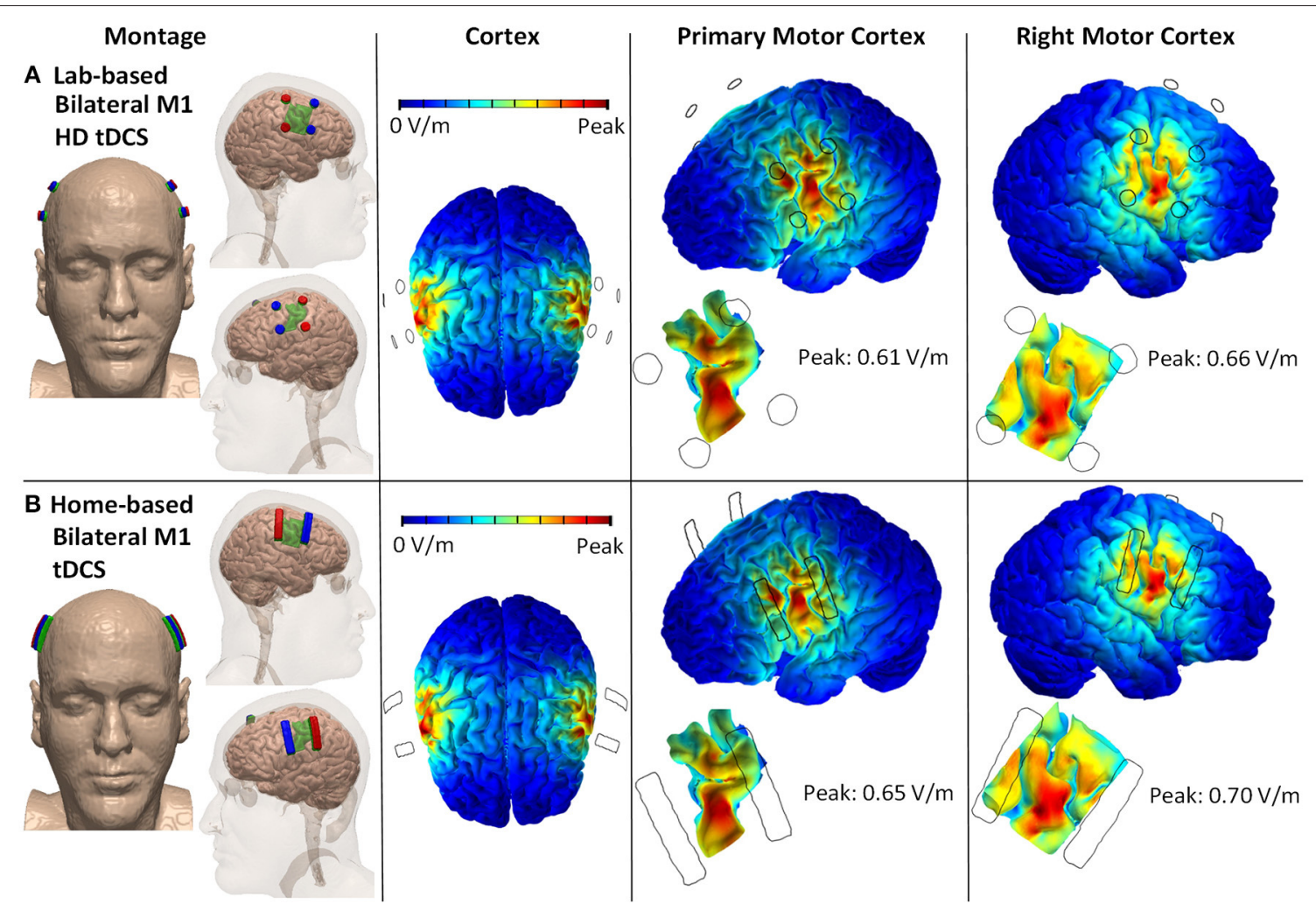

FIGURE 1 | Lab- and home-based bilateral M1 HD-tDCS montages and computational modeling on 3D rendered head built from the MRI derived segmentation masks. (A) Lab-based with anode (red) placed over C3/C5 and cathode (blue) over FC3/FC5. (B) Home-based (strip) covering same regions.

\section{COMPUTATIONAL MODELS AND MODEL-DRIVEN DESIGN OF NEW IDEA}

\section{Computational Modeling of Bilateral M1 HD-TDCS}

An individualized high-resolution finite element (FE) head model was developed from the T1-weighted MRI scan $\left(1 \mathrm{~mm}^{3}\right)$ of an adult healthy male using an automated segmentation algorithm within Statistical Parametric Mapping (SPM8, Wellcome Centre for Human Neuroimaging, London, UK). Additionally, in-house MATLAB scripts (ROAST) were used to smooth artifacts and remove discontinuities from the six different segmented image masks generated within SPM8 (103). Manual segmentation was then performed in ScanIP (Simpleware Ltd, Exeter, UK) to separate out fat from the automatically segmented image mask of skin. Stimulation electrodes, sponge pads, and gels were modeled in SolidWorks (Dassault Systèmes Corp., Waltham, MA) and imported into ScanIP for meshing. The meshes were then imported into an FE package (COMSOL Multiphysics 4.3, COMSOL, Inc., Burlington, MA, USA). Following isotropic electrical conductivities were assigned to the corresponding tissue layers and electrodes in S/m: skin: 0.465 , Fat: 0.025 , skull: 0.01 , CSF: 0.85 , gray matter: 0.276 , white matter: 0.126 , air: $10^{-15}$, electrode: $5.99 \times 10^{7}$, and conductive gel/sponge pad: 1.4. Laplace equation $(\nabla \cdot(\sigma \nabla V)=0)$ was solved (104) and boundary conditions were used such that the current density corresponding to $2 \mathrm{~mA}$ of total current was applied at the anode(s) on each side (left/right M1 cortices). The ground was applied to the cathode(s). The FEM model was used to predict and compare the cortical brain current flow patterns between two different stimulation montages, $12 \mathrm{~mm} H \mathrm{HD}$ disk (Lab-based bilateral excitation-primary and right motor cortex) and $4 \mathrm{~cm} \times 1 \mathrm{~cm}$ electrode strip (Home-based bilateral excitation-primary and right motor cortex). Our analyses here address current targeting, and the technology we propose can be implemented with a selection of intensity and duration, and also be adapted for transcranial alternating current stimulation (tACS) or other frequency based approaches.

\section{Lab-Based Bilateral HD-TDCS $2 \times 2$}

Twelve millimeter diameter disk electrodes with two anodes and two cathodes positioned posterior to anterior across the face/head region of each the left and right motor cortex. Explicit 10-10 locations for the anodes are C3, C5, C4, C6, and for the cathodes are FC3, FC4, FC5, FC6 (Figure 1A).

\section{Home-Based Bilateral TDCS $2 \times 2$}

$4 \mathrm{~cm} \times 1 \mathrm{~cm}$ strip electrodes with two anodes and two cathodes positioned posterior to anterior across the face/head region of the primary and right motor cortex. Anodes are positioned 

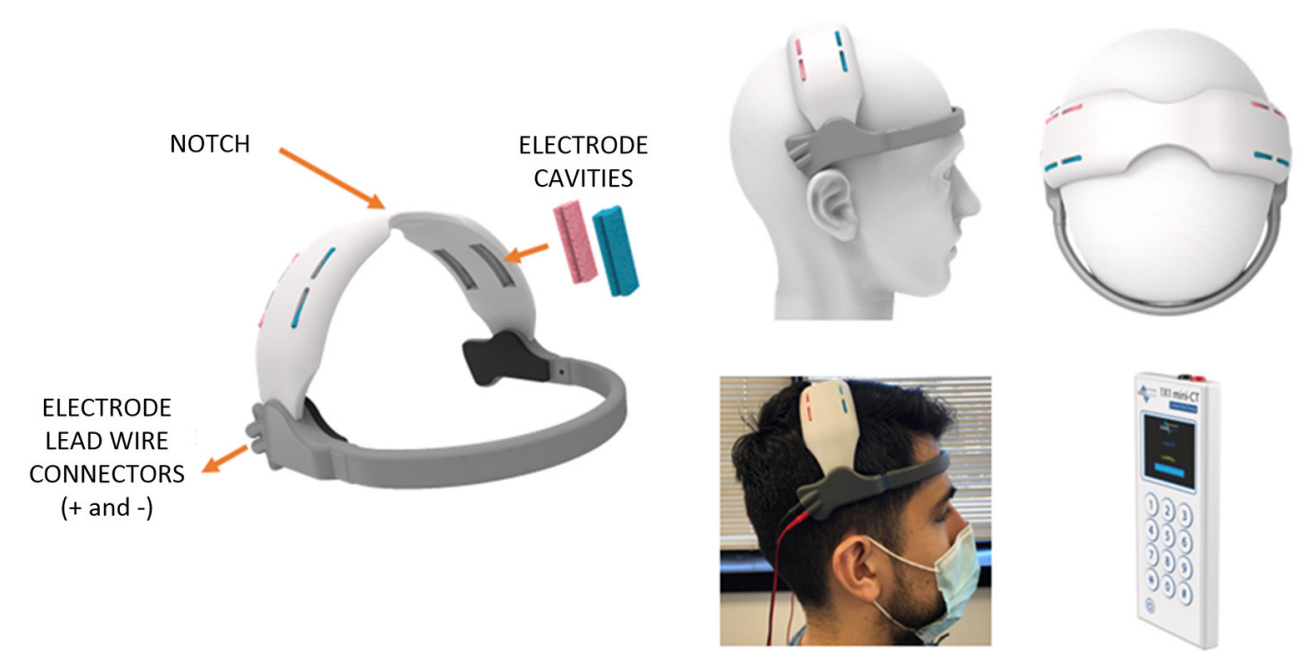

FIGURE 2 | Model-driven design of home-based HD-tDCS application for bilateral M1 stimulation.

horizontally across C3 and C5 and across C4 and C6. Cathodes are positioned horizontally across FC3 and FC5 and across FC4 and FC6 (Figure 1B).

The main sources for variability in the peak electric field between the two stimulation montages are differences in electrode dimensions. Home-based montage uses $4 \mathrm{~cm} \times 1 \mathrm{~cm}$ strip electrodes, whereas the lab-based montage uses $12 \mathrm{~mm}$ diameter disk electrodes. The conductivity of variables (skin, fat, skull, CSF, gray matter, white matter, air, electrode, and conductive gel/sponge pad) does not vary across the two stimulation montages.

Generally, a focality of targeting or applied current intensity impacts electric field intensities. Inter-individual differences, including anatomical and functional brain state, appeared to affect the variability in the current flow in addition to the varying levels of tDCS dose (e.g., montages, current intensity) (105) thus should be understood primarily to achieve optimal outcomes.

\section{Development of a Home-Based HD-TDCS Headset}

We pursued a typical iterative industrial design workflow beginning first with finalizing design constraints of the envisioned self-administered home headset. The critical functional specifications included: (1) use of a stimulation electrode solution meeting size requirement $(4 \mathrm{~cm} \times 1 \mathrm{~cm})$, (2) need for a disposable electrode, (3) maintenance of a short anode to cathode electrode separation (replicating the distance assumed in computational model) while preventing unintended shorting, and (4) option for multiple electrode loading slots corresponding to the motor homunculus. Additionally, design requirements related to fit, usability, and aesthetics were incorporated such as secure loading, maintenance of electrode-scalp contact, subject comfort, ease of administration, and concealing of electrode leadwires. A sketch ideation phase realizing stated design constraints was pursued followed by 3D Computer Aided Design (CAD) modeling (SolidWorks, Dassault Systems SolidWorks Corp, USA). This was followed by 3D printing and associated machining to verify early concepts. These aforementioned steps were repeated until headset design was considered suitable for proceeding to the final step-i.e., suitable for loading on actual human subjects. The final design as depicted in Figure 2 consists of a forehead and a motor band. The electrode lead wires from the stimulation device are connected to the two connectors at the end of the forehead band. The electrode wires are routed within the motor band to provide the needed concealment. The forehead band includes cushioning material at different sections to help with fit and comfort. The motor band includes cavities to hold the saline-soaked single-use (disposable) sponge strips covering different levels of the homunculus. A notch is included in the center to help the user align with the nasion during the self-application process (106). A conductive carbon electrode strip is held at the base of the cavity to make the electrical connection to the sponge. Prototypes were printed using a Form 2 SLA printer (Formlabs, MA, USA) at a layer thickness of 100 microns. Though subject to feasibility clinical trials, a further principle advantage of our approach is placing electrodes across M1 will naturally drive current to (and maximize intensity at) motor cortex.

\section{CONCLUSION}

Taken together, our suggested home-based HD-tDCS with enhanced focality over bilateral M1 is a highly feasible approach enabling multiple sessions at home. This claim is supported by the observation that tDCS effects are cumulative, and thus, consecutive stimulation spanning weeks to months would induce a longer-lasting impact on the neural plasticity which 
tDCS intends to modulate. In this manner, our new approach paves the way for novel mechanism-based treatments utilizing neuromodulation and telehealth while satisfying the compelling needs of patients and their families.

\section{AUTHOR CONTRIBUTIONS}

AFD, AD, and JS: design and development of home-based tDCS and analysis of data. AFD, $\mathrm{AD}$, JS, DK, and MB: drafting the

\section{REFERENCES}

1. Nijs J, George SZ, Clauw DJ, Fernández-de-las-Peñas C, Kosek E, Ickmans K, et al. Central sensitisation in chronic pain conditions: latest discoveries and their potential for precision medicine. Lancet Rheumatol. (2021) 3:e383-e92. doi: 10.1016/S2665-9913(21)00032-1

2. Burstein R, Jakubowski M, Garcia-Nicas E, Kainz V, Bajwa Z, Hargreaves $\mathrm{R}$, et al. Thalamic sensitization transforms localized pain into widespread allodynia. Ann Neurol. (2010) 68:81-91. doi: 10.1002/ana.21994

3. Cohen SP, Vase L, Hooten WM. Chronic pain: an update on burden, best practices, new advances. Lancet. (2021) 397:2082-97. doi: 10.1016/S0140-6736(21)00393-7

4. Nicholas M, Vlaeyen JWS, Rief W, Barke A, Aziz Q, Benoliel R, et al. The IASP classification of chronic pain for ICD-11: chronic primary pain. Pain. (2019) 160:28-37. doi: 10.1097/j.pain.0000000000001390

5. Fitzcharles, M.-A., Cohen SP, Clauw DJ, Littlejohn G, Usui C, et al. Nociplastic pain: towards an understanding of prevalent pain conditions. Lancet. (2021) 397:2098-110. doi: 10.1016/S0140-6736(21)00392-5

6. Olesen J, Friberg L, Olsen TS, Iversen HK, Lassen NA, Andersen AR, et al. Timing and topography of cerebral blood flow, aura, and headache during migraine attacks. Ann Neurol. (1990) 28:791-8. doi: 10.1002/ana.410280610

7. Barbanti P, Fofi L, Grazzi L, Vernieri F, Camarda C, Torelli P, et al. Clinical features, disease progression, and use of healthcare resources in a large sample of 866 patients from 24 headache centers: A real-life perspective from the Italian chronic migraine (IRON) project. Headache J Head Face Pain. (2021) 61:936-50. doi: 10.1111/HEAD.14123

8. Herman PM, Broten N, Lavelle TA, Sorbero ME, Coulter ID. Healthcare costs and opioid use associated with high-impact chronicspinal pain in the United States. Spine. (2019) 44:1154. doi: 10.1097/BRS.0000000000003033

9. Jantarada C, Silva C, Guimarães-Pereira L. Prevalence of problematic use of opioids in patients with chronic noncancer pain: a systematic review with meta-analysis. Pain Pract. (2021) 21:715-29. doi: 10.1111/papr.13001

10. DosSantos MF, Ferreira N, Toback RL, Carvalho AC, DaSilva AF. Potential mechanisms supporting the value of motor cortex stimulation to treat chronic pain syndromes. Front Neurosci. (2016) 10:18. doi: 10.3389/fnins.2016.00018

11. Bikson M, Brunoni AR, Charvet LE, Clark VP, Cohen LG, Deng Z, et al. Rigor and reproducibility in research with transcranial electrical stimulation: an NIMH-sponsored workshop. Brain Stimul. (2018) 11:465-80. doi: 10.1016/j.brs.2017.12.008

12. DaSilva AF, Volz MS, Bikson M, Fregni F. Electrode positioning and montage in transcranial direct current stimulation. J V Exp. (2011) 51:e2744. doi: $10.3791 / 2744$

13. Minhas P, Bansal V, Patel J, Ho JS, Diaz J, Datta A, et al. Electrodes for high-definition transcutaneous DC stimulation for applications in drug delivery and electrotherapy, including tDCS. $J$ Neurosci Methods. (2010) 190:188-97. doi: 10.1016/j.jneumeth.2010. 05.007

14. Villamar MF, Volz MS, Bikson M, Datta A, Dasilva AF, Fregni F. Technique and considerations in the use of $4 \times 1$ ring high-definition transcranial direct current stimulation (HD-tDCS). J Vis Exp. (2013) 77:e50309. doi: $10.3791 / 50309$ manuscript. All authors contributed to the article, revision for intellectual content, and approved the submitted version.

\section{FUNDING}

This study was supported by grants from the National Institutes of Health - National Institute of Neurological Disorders and Stroke (NIH-NINDS R01 NS094413) and National Institute of Dental and Craniofacial Research (NIH-NIDCR U01 DE025633) United States (AD).

15. Caparelli-Daquer EM, Zimmermann TJ, Mooshagian E, Parra LC, Rice JK, Datta A, et al. A pilot study on effects of $4 \times 1$ High-Definition tDCS on motor cortex excitability. Proc Annu Int Conf IEEE Eng Med Biol Soc. (2012) 2012:735-8. doi: 10.1109/EMBC.2012.6346036

16. Kuo HI, Bikson M, Datta A, Minhas P, Paulus W, Kuo MF, et al. Comparing cortical plasticity induced by conventional and high-definition $4 \times 1$ ring tDCS: a neurophysiological study. Brain Stimul. (2013) 6:644-8. doi: 10.1016/j.brs.2012.09.010

17. Donnell A, Nascimento TD, Lawrence M, Gupta V, Zieba T, Truong $\mathrm{DQ}$, et al. High-definition and non-invasive brain modulation of pain and motor dysfunction in chronic TMD. Brain Stimul. (2015) 8:1085-92. doi: 10.1016/j.brs.2015.06.008

18. Bikson M, Esmaeilpour Z, Adair D, Kronberg G, Tyler WJ, Antal A, et al. Transcranial electrical stimulation nomenclature. Brain Stimul. (2019) 12:1349-66. doi: 10.1016/j.brs.2019.07.010

19. Tsubokawa T, Katayama Y, Yamamoto T, Hirayama T, Koyama S. Treatment of thalamic pain by chronic motor cortex stimulation. Pacing Clin Electrophysiol. (1991) 14:131-4. doi: 10.1111/j.1540-8159.1991.tb04058.x

20. Kishima H, Saitoh Y, Osaki Y, Nishimura H, Kato A, Hatazawa J, et al. Motor cortex stimulation in patients with deafferentation pain: activation of the posterior insula and thalamus. J Neurosurg. (2007) 107:43-8. doi: 10.3171/JNS-07/07/0043

21. Rodríguez RF, Contreras N. Bilateral motor cortex stimulation for the relief of central dysesthetic pain and intentional tremor secondary to spinal cord surgery: a case report. Neuromodulation Technol Neural Interface. (2002) 5:189-95. doi: 10.1046/j.1525-1403.2002.02029.x

22. Tani N, Saitoh Y, Hirata M, Kato A, Yoshimine T. Bilateral cortical stimulation for deafferentation pain after spinal cord injury: case report. $J$ Neurosurg. (2004) 101:687-9. doi: 10.3171/jns.2004.101.4.0687

23. Shehata HS, Esmail EH, Abdelalim A, El-Jaafary S, Elmazny A, Sabbah A, et al. Repetitive transcranial magnetic stimulation versus botulinum toxin injection in chronic migraine prophylaxis: a pilot randomized trial. J Pain Res. (2016) 9:771. doi: 10.2147/JPR.S116671

24. Zardouz S, Shi L, Leung A. A feasible repetitive transcranial magnetic stimulation clinical protocol in migraine prevention. SAGE Open Med Case Rep. (2016) 4:2050313X16675257. doi: 10.1177/2050313X16675257

25. Misra UK, Kalita J, Tripathi G, Bhoi SK. Role of $\beta$ endorphin in pain relief following high rate repetitive transcranial magnetic stimulation in migraine. Brain Stimul. (2017) 10:618-23. doi: 10.1016/j.brs.2017.02.006

26. Tripathi GM, Kalita J, Misra UK. Role of glutamate and its receptors in migraine with reference to amitriptyline and transcranial magnetic stimulation therapy. Brain Res. (2018) 1696:31-7. doi: 10.1016/j.brainres.2018.05.046

27. Hodaj H, Alibeu JP, Payen JF, Lefaucheur JP. Treatment of chronic facial pain including cluster headache by repetitive transcranial magnetic stimulation of the motor cortex with maintenance sessions: a naturalistic study. Brain Stimul. (2015) 8:801-7. doi: 10.1016/j.brs.2015.01.416

28. Leung A, Fallah A, Shukla S, Lin L, Tsia A, Song D, et al. Case series rTMS in alleviating mild TBI related headaches-a case series. Pain Physician. (2016) 19:347-53. doi: 10.36076/ppj/2016.19.E347

29. Quesada C, Pommier B, Fauchon C, Bradley C, Créac'h C, Vassal F, et al. Robot-guided neuronavigated repetitive transcranial magnetic stimulation 
(rTMS) in central neuropathic pain. Arch. Phys. Med. Rehabil. (2018) 99:2203-15.e1. doi: 10.1016/j.apmr.2018.04.013

30. Lazzaro V, Dileone M, Pilato F, Contarino M-F, Musumeci G, Bentivoglio $\mathrm{AR}$, et al. Bentivoglio AR, et al. Repetitive transcranial magnetic stimulation of the motor cortex for hemichorea. J. Neurol. Neurosurg. Psychiatry. (2006) 77:1095-7. doi: 10.1136/jnnp.2005.082875

31. Kang BS, Shin HI, Bang MS. Effect of repetitive transcranial magnetic stimulation over the hand motor cortical area on central pain after spinal cord injury. Arch Phys Med Rehabil. (2009) 90:1766-71. doi: 10.1016/j.apmr.2009.04.008

32. Mhalla A, Baudic S, De Andrade DC, Gautron M, Perrot S, Teixeira MJ, et al. Long-term maintenance of the analgesic effects of transcranial magnetic stimulation in fibromyalgia. PAIN. (2011) 152:1478-85. doi: 10.1016/j.pain.2011.01.034

33. Lee SJ, Kim DY, Chun MH, Kim YG. The effect of repetitive transcranial magnetic stimulation on fibromyalgia: a randomized sham-controlled trial with 1-mo follow-up. Am J Phys Med Rehabil. (2012) 91:1077-85. doi: 10.1097/PHM.0b013e3182745a04

34. Irlbacher K, Kuhnert J, Röricht S, Meyer BU, Brandt SA. Zentrale und periphere Deafferenzierungsschmerzen. Der Nervenarzt. (2006) 77:1196203. doi: $10.1007 / \mathrm{s} 00115-006-2148-1$

35. Lefaucheur, J.-P., Drouot X, Menard-Lefaucheur I, Zerah F, Bendib B, et al. Neurogenic pain relief by repetitive transcranial magnetic cortical stimulation depends on the origin and the site of pain. J Neurol Neurosurg Psychiatry. (2004) 75:612-6. doi: 10.1136/jnnp.2003.022236

36. Di Rollo A, Pallanti S. Phantom limb pain: low frequency repetitive transcranial magnetic stimulation in unaffected hemisphere. Case Rep Med. (2011) 2011:130751. doi: 10.1155/2011/130751

37. André-Obadia N, Peyron R, Mertens P, Mauguière F, Laurent B, GarciaLarrea L. Transcranial magnetic stimulation for pain control. Doubleblind study of different frequencies against placebo, and correlation with motor cortex stimulation efficacy. Clin Neurophysiol. (2006) 117:1536-44. doi: 10.1016/j.clinph.2006.03.025

38. Defrin R, Grunhaus L, Zamir D, Zeilig G. The effect of a series of repetitive transcranial magnetic stimulations of the motor cortex on central pain after spinal cord injury. Arch Phys Med Rehabil. (2007) 88:1574-80. doi: 10.1016/j.apmr.2007.07.025

39. Kobayashi M, Fujimaki T, Mihara B, Ohira T. Repetitive transcranial magnetic stimulation once a week induces sustainable long-term relief of central poststroke pain. Neuromodulation Technol Neural Interface. (2015) 18:249-54. doi: 10.1111/ner.12301

40. Saitoh Y, Hirayama A, Kishima H, Shimokawa T, Oshino S, Hirata M, et al. Reduction of intractable deafferentation pain due to spinal cord or peripheral lesion by high-frequency repetitive transcranial magnetic stimulation of the primary motor cortex. J Neurosurg. (2007) 107:555-9. doi: $10.3171 /$ JNS-07/09/0555

41. Onesti E, Gabriele M, Cambieri C, Ceccanti M, Raccah R, Stefano G, et al. H-coil repetitive transcranial magnetic stimulation for pain relief in patients with diabetic neuropathy. Eur J Pain. (2013) 17:1347-56. doi: $10.1002 / j .1532-2149.2013 .00320 . x$

42. Shimizu T, Hosomi K, Maruo T, Goto Y, Yokoe M, Kageyama Y, et al. Efficacy of deep rTMS for neuropathic pain in the lower limb: a randomized, doubleblind crossover trial of an H-coil and figure-8 coil. J Neurosurg. (2017) 127:1172-80. doi: 10.3171/2016.9.JNS16815

43. Fregni F, Boggio PS, Lima MC, Ferreira MJL, Wagner T, Rigonatti SP, et al. A sham-controlled, phase II trial of transcranial direct current stimulation for the treatment of central pain in traumatic spinal cord injury. Pain. (2006) 122:197-209. doi: 10.1016/j.pain.2006.02.023

44. Ahn H, Woods AJ, Kunik ME, Bhattacharjee A, Chen Z, Choi E, et al. Efficacy of transcranial direct current stimulation over primary motor cortex (anode) and contralateral supraorbital area (cathode) on clinical pain severity and mobility performance in persons with knee osteoarthritis: an experimenterand participant-blinded, randomized, sham-controlled pilot clinical study. Brain Stimul. (2017) 10:902-9. doi: 10.1016/j.brs.2017.05.007

45. Fregni F, Gimenes R, Valle AC, Ferreira MJL, Rocha RR, Natalle L, et al. A randomized, sham-controlled, proof of principle study of transcranial direct current stimulation for the treatment of pain in fibromyalgia. Arthritis Rheum. (2006) 54:3988-98. doi: 10.1002/art.22195
46. DaSilva AF, Mendonca ME, Zaghi S, Lopes M, DosSantos MF, Spierings EL, et al. tDCS-induced analgesia and electrical fields in pain-related neural networks in chronic migraine. Headache J Head Face Pain. (2012) 52:128395. doi: 10.1111/j.1526-4610.2012.02141.x

47. Przeklasa-Muszyńska A, Kocot-Kepska M, Dobrogowski J, Wiatr M, Mika J. Transcranial direct current stimulation (tDCS) and its influence on analgesics effectiveness in patients suffering from migraine headache. Pharmacol Rep. (2017) 69:714-21. doi: 10.1016/j.pharep.2017.02.019

48. Molero-Chamizo A, Salas Sánchez Á, Álvarez Batista B, Cordero García C, Andújar Barroso R, Rivera-Urbina GN, et al. Bilateral motor cortex tDCS effects on post-stroke pain and spasticity: a three cases study. Front Pharmacol. (2021) 12:624582. doi: 10.3389/fphar.2021.624582

49. Villamar MF, Wivatvongvana P, Patumanond J, Bikson M, Truong DQ, Datta A, et al. Focal modulation of the primary motor cortex in fibromyalgia using $4 \times 1$-ring high-definition transcranial direct current stimulation (HDtDCS): immediate and delayed analgesic effects of cathodal and anodal stimulation. J Pain. (2013) 14:371-83. doi: 10.1016/j.jpain.2012.12.007

50. Castillo-Saavedra L, Gebodh N, Bikson M, Diaz-Cruz C, Brandao R, Coutinho L, et al. Clinically effective treatment of fibromyalgia pain with high-definition transcranial direct current stimulation: phase ii open-label dose optimization. J Pain. (2016) 17:14-26. doi: 10.1016/j.jpain.2015.09.009

51. Nitsche MA, Paulus W. Excitability changes induced in the human motor cortex by weak transcranial direct current stimulation. J Physiol. (2000) 527:633. doi: 10.1111/j.1469-7793.2000.t01-1-00633.x

52. Agboada D, Mosayebi-Samani M, Kuo MF, Nitsche MA. Induction of longterm potentiation-like plasticity in the primary motor cortex with repeated anodal transcranial direct current stimulation - Better effects with intensified protocols? Brain Stimul. (2020) 13:987-97. doi: 10.1016/j.brs.2020.04.009

53. Feng Y, Zhang B, Zhang J, Yin Y. Effects of non-invasive brain stimulation on headache intensity and frequency of headache attacks in patients with migraine: a systematic review and meta-analysis. Headache. (2019) 59:143647. doi: 10.1111/head.13645

54. Cai G, Xia Z, Charvet L, Xiao F, Datta A, Androulakis XM. A systematic review and meta-analysis on the efficacy of repeated transcranial direct current stimulation for migraine. J Pain Res. (2021) 14:1171-83. doi: $10.2147 /$ JPR.S295704

55. Silva G, Miksad R, Freedman SD, Pascual-Leone A, Jain S, Gomes DL, et al. Treatment of cancer pain with noninvasive brain stimulation. J Pain Symptom Manage. (2007) 34:342-5. doi: 10.1016/j.jpainsymman.2007.06.002

56. Hu, X.-S., Fisher CA, Munz SM, Toback RL, Nascimento TD, et al. Feasibility of non-invasive brain modulation for management of pain related to chemoradiotherapy in patients with advanced head and neck cancer. Front Hum Neurosci. (2016) 10:466. doi: 10.3389/fnhum.2016. 00466

57. Ibrahim NM, Abdelhameed KM, Kamal SMM, Khedr EMH, Kotb HIM. Effect of transcranial direct current stimulation of the motor cortex on visceral pain in patients with hepatocellular carcinoma. Pain Med. (2018) 19:550-60. doi: 10.1093/pm/pnx087

58. DosSantos MF, Love TM, Martikainen IK, Nascimento TD, Fregni F, Cummiford C, et al. Immediate effects of tDCS on the $\mu$-opioid system of a chronic pain patient. Front Psychiatry. (2012) 3:93. doi: $10.3389 /$ fpsyt.2012.00093

59. Borckardt JJ, Reeves ST, Robinson SM, May JT, Epperson TI, Gunselman RJ, et al. Transcranial direct current stimulation (tDCS) reduces postsurgical opioid consumption in total knee arthroplasty (TKA). Clin J Pain. (2013) 29:925-28. doi: 10.1097/AJP.0b013e31827e32be

60. Glaser J, Reeves ST, Stoll WD, Epperson TI, Hilbert M, Madan A, et al. Motor/prefrontal transcranial direct current stimulation (tDCS) following lumbar surgery reduces postoperative analgesia use. Spine. (2016) 41:835-9. doi: $10.1097 /$ BRS.0000000000001525

61. Valle A, Roizenblatt S, Botte S, Zaghi S, Riberto M, Tufik S, et al. Efficacy of anodal transcranial direct current stimulation (tDCS) for the treatment of fibromyalgia: Results of a randomized, sham-controlled longitudinal clinical trial. J Pain Manag. (2009) 2:353-61. Available online at: http://www. novapublishers.org/catalog/product_info.php?products_id=51069

62. O'Connell NE, Marston L, Spencer S, DeSouza LH, Wand BM. Non-invasive brain stimulation techniques for chronic pain. Cochrane Database Syst Rev. (2018) 4:CD008208. doi: 10.1002/14651858.CD008208.pub4 
63. Sachs AJ, Babu H, Su Y-F, Miller KJ, Henderson JM. Lack of efficacy of motor cortex stimulation for the treatment of neuropathic pain in 14 patients. Neuromodulation Technol Neural Interface. (2014) 17:303-11. doi: $10.1111 /$ ner. 12181

64. Fontaine D, Hamani C, Lozano A. Efficacy and safety of motor cortex stimulation for chronic neuropathic pain: critical review of the literature: clinical article. J Neurosurg. (2009) 110:251-6. doi: 10.3171/2008.6. 17602

65. Mo J-J, Hu W-H, Zhang C, Wang X, Liu C, Zhao B-T, et al. Motor cortex stimulation: a systematic literature-based analysis of effectiveness and case series experience. BMC Neurol. (2019) 19:48. doi: 10.1186/s12883-019-1273-y

66. Ziemann U, Paulus W, Nitsche MA, Pascual-Leone A, Byblow WD, Berardelli A, et al. Consensus: motor cortex plasticity protocols. Brain Stimul. (2008) 1:164-82. doi: 10.1016/j.brs.2008.06.006

67. Barker AT, Jalinous R, Freeston IL. Non-invasive magnetic stimulation of human motor cortex. Lancet. (1985) 325:1106-7. doi: 10.1016/S0140-6736(85)92413-4

68. Siebner HR, Tormos JM, Ceballos-Baumann AO, Auer C, Catala MD, Conrad B, et al. Low-frequency repetitive transcranial magnetic stimulation of the motor cortex in writer's cramp. Neurology. (1999) 52:529. doi: 10.1212/WNL.52.3.529

69. Siebner H, Rothwell J. Transcranial magnetic stimulation: new insights into representational cortical plasticity. Exp Brain Res. (2002) 148:1-16. doi: 10.1007/s00221-002-1234-2

70. Pell GS, Roth Y, Zangen A. Modulation of cortical excitability induced by repetitive transcranial magnetic stimulation: influence of timing and geometrical parameters and underlying mechanisms. Prog Neurobiol. (2011) 93:59-98. doi: 10.1016/j.pneurobio.2010.10.003

71. Cohen LG, Roth BJ, Nilsson J, Dang N, Panizza M, Bandinelli S, et al. Effects of coil design on delivery of focal magnetic stimulation. Technical considerations. Electroencephalogr Clin. Neurophysiol. (1990) 75:350-7. doi: 10.1016/0013-4694(90)90113-X

72. Dmochowski JP, Datta A, Bikson M, Su Y, Parra LC. Optimized multielectrode stimulation increases focality and intensity at target. J Neural Eng. (2011) 8:046011. doi: 10.1088/1741-2560/8/4/046011

73. DaSilva AF, Truong DQ, DosSantos MF, Toback RL, Datta A, Bikson M. State-of-art neuroanatomical target analysis of high-definition and conventional tDCS montages used for migraine and pain control. Front Neuroanat. (2015) 9:89. doi: 10.3389/fnana.2015.00089

74. Borckardt JJ, Bikson M, Frohman H, Reeves ST, Datta A, Bansal V, et al. A pilot study of the tolerability and effects of high-definition transcranial direct current stimulation (HD-tDCS) on pain perception. J Pain. (2012) 13:112-20. doi: 10.1016/j.jpain.2011.07.001

75. da Silva Machado DG, Bikson M, Datta A, Caparelli-Dáquer E, Unal G, Baptista AF, et al. Acute effect of high-definition and conventional tDCS on exercise performance and psychophysiological responses in endurance athletes: a randomized controlled trial. Sci Rep. (2021) 11:1-15. doi: 10.1038/s41598-021-92670-6

76. Datta A, Truong D, Minhas P, Parra LC, Bikson M. Inter-individual variation during transcranial direct current stimulation and normalization of dose using MRI-derived computational models. Front Psychiatry. (2012) 3:91. doi: 10.3389/fpsyt.2012.00091

77. Edwards D, Cortes M, Datta A, Minhas P, Wassermann EM, Bikson M. Physiological and modeling evidence for focal transcranial electrical brain stimulation in humans: a basis for high-definition tDCS. Neuroimage. (2013) 74:266-75. doi: 10.1016/j.neuroimage.2013.01.042

78. Rawji V, Ciocca M, Zacharia A, Soares D, Truong D, Bikson M, et al. tDCS changes in motor excitability are specific to orientation of current flow. Brain Stimul. (2018) 11:289-98. doi: 10.1016/j.brs.2017.11.001

79. Hannah R, Iacovou A, Rothwell JC. Direction of TDCS current flow in human sensorimotor cortex influences behavioural learning. Brain Stimul. (2019) 12:684-92. doi: 10.1016/j.brs.2019.01.016

80. Kaciroti N, DosSantos MF, Moura B, Bellile EL, Nascimento TD, Maslowski E, et al. Sensory-Discriminative three-dimensional body pain mobile app measures versus traditional pain measurement with a visual analog scale: validation study. JMIR mHealth uHealth. (2020) 8:e17754. doi: $10.2196 / 17754$
81. Lefaucheur JP, Antal A, Ayache SS, Benninger DH, Brunelin J, Cogiamanian $\mathrm{F}$, et al. Evidence-based guidelines on the therapeutic use of transcranial direct current stimulation (tDCS). Clin Neurophysiol. (2017) 128:56-92. doi: 10.1016/j.clinph.2016.10.087

82. Stilling JM, Monchi O, Amoozegar F, Debert CT. Transcranial Magnetic and Direct Current Stimulation (TMS/tDCS) for the treatment of headache: a systematic review. Headache J Head Face Pain. (2019) 59:339-57. doi: 10.1111/head.13479

83. Fregni F, El-Hagrassy MM, Pacheco-Barrios K, Carvalho S, Leite J, Simis $\mathrm{M}$, et al. Evidence-based guidelines and secondary meta-analysis for the use of transcranial direct current stimulation in neurological and psychiatric disorders. Int J Neuropsychopharmacol. (2021) 24:256. doi: 10.1093/ijnp/pyaa051

84. Maezawa H, Vicario CM, Kuo M-F, Hirata M, MimaT, Nitsche MA. Effects of bilateral anodal transracial direct current stimulation over the tongue primary motor cortex on cortical excitability of the tongue and tongue motor functions. Brain Stimul BasicTransl Clin Res Neuromodulation. (2020) 13:270-2. doi: 10.1016/J.BRS.2019.10.005

85. Gregoret L, Zamorano AM, Graven-Nielsen T. Effects of multifocal transcranial direct current stimulation targeting the motor network during prolonged experimental pain. Eur J Pain. (2021) 25:1241-53. doi: 10.1002/ejp.1743

86. DaSilva AF, Nascimento TD, DosSantos MF, Zubieta JK. Migraine and the mu-opioidergic system - Can we directly modulate it? Evidence from neuroimaging studies. Curr Pain Headache Rep. (2014) 18:429. doi: 10.1007/s11916-014-0429-0

87. Foerster BR, Nascimento TD, Deboer M, Clauw DJ, Zubieta J, Harris RE. Excitatory and inhibitory brain matabolites as targets and predictors of effective motor cortex tDCS therapy in fibromyalgia. Arthritis Rheumatol. (2015) 67:576-81. doi: 10.1002/art.38945

88. Cummiford CM, Nascimento TD, Foerster BR, Clauw DJ, Zubieta J$\mathrm{K}$, Harris RE, et al. Changes in resting state functional connectivity after repetitive transcranial direct current stimulation applied to motor cortex in fibromyalgia patients. Arthritis Res Ther. (2016) 18:40. doi: 10.1186/s13075-016-0934-0

89. Naegel S, Biermann J, Theysohn N, Kleinschnitz C, Diener HC, Katsarava Z, et al. Polarity-specific modulation of pain processing by transcranial direct current stimulation - a blinded longitudinal fMRI study. J Headache Pain. (2018) 19:1-10. doi: 10.1186/s10194-0180924-5

90. Meeker TJ, Keaser ML, Khan SA, Gullapalli RP, Seminowicz DA, Greenspan JD. Non-invasive motor cortex neuromodulation reduces secondary hyperalgesia and enhances activation of the descending pain modulatory network. Front Neurosci. (2019) 13:467. doi: 10.3389/fnins.2019. 00467

91. Lim M, Kim DJ, Nascimento TD, Ichesco E, Kaplan C, Harris RE, et al. Functional magnetic resonance imaging signal variability is associated with neuromodulation in fibromyalgia. Neuromodulation Technol Neural Interface. (2021). doi: 10.1111/ner.13512. [Epub ahead of print].

92. Harte SE, Harris RE, Clauw DJ. The neurobiology of central sensitization. J Appl Biobehav Res. (2018) 23:e12137. doi: 10.1111/jabr.12137

93. Cosentino G, Brighina F, Talamanca S, Paladino P, Vigneri S, Baschi R, et al. Reduced threshold for inhibitory homeostatic responses in migraine motor cortex? A tDCS/TMS Study. Headache J Head Face Pain. (2014) 54:663-74. doi: 10.1111/head.12249

94. Wickmann F, Stephani C, Czesnik D, Klinker F, Timäus C, Chaieb $\mathrm{L}$, et al. Prophylactic treatment in msenstrual migraine: a proof-ofconcept study. J Neurol Sci. (2015) 354:103-9. doi: 10.1016/j.jns.2015. 05.009

95. De Icco R, Putort,ì A, De Paoli I, Ferrara E, Cremascoli R, Terzaghi $\mathrm{M}$, et al. Anodal transcranial direct current stimulation in chronic migraine and medication overuse headache: a pilot double-blind randomized sham-controlled trial. Clin Neurophysiol. (2021) 132:126-36. doi: 10.1016/j.clinph.2020.10.014

96. Andr,é S, Heinrich S, Kayser F, Menzler K, Kesselring J, Khader PH, et al. Athome tDCS of the left dorsolateral prefrontal cortex improves visual shortterm memory in mild vascular dementia. J Neurol Sci. (2016) 369:185-90. doi: 10.1016/j.jns.2016.07.065 
97. Hyvärinen P, Mäkitie A, Aarnisalo AA. Self-administered domiciliary tDCS treatment for tinnitus: a double-blind sham-controlled study. PLoS One. (2016) 11:e0154286. doi: 10.1371/journal.pone.01 54286

98. Charvet L, Shaw M, Dobbs B, Frontario A, Sherman K, Bikson M. et al. (2018). Remotely Supervised Transcranial Direct Current Stimulation Increases the Benefit of At-Home Cognitive Training in Multiple Sclerosis. Neuromodulation. 21:383-9. doi: 10.1111/ner.12583

99. Brietzke AP, Zortea M, Carvalho F, Sanches PRS, Silva DPJ, Torres IL, et al. Large treatment effect with extended home-based transcranial direct current stimulation over dorsolateral prefrontal cortex in fibromyalgia: a proof of concept sham-randomized clinical study. J Pain. (2020) 21:212-24. doi: 10.1016/j.jpain.2019.06.013

100. Ahn H, Sorkpor S, Miao H, Zhong C, Jorge R, Park L, et al. Home-based self-administered transcranial direct current stimulation in older adults with knee osteoarthritis pain: an open-label study. J Clin Neurosci. (2019) 66:61-5. doi: 10.1016/j.jocn.2019.05.023

101. Riggs A, Patel V, Paneri B, Portenoy RK, Bikson M, Knotkova H. At-home transcranial direct current stimulation (tDCS) with telehealth support for symptom control in chronically-ill patients with multiple symptoms. Front Behav Neurosci. (2018) 12:93. doi: 10.3389/fnbeh.2018. 00093

102. Charvet LE, Shaw MT, Bikson M, Woods AJ, Knotkova H. Supervised transcranial direct current stimulation (tDCS) at home: a guide for clinical research and practice. Brain Stimul. (2020) 13:686-93. doi: 10.1016/j.brs.2020.02.011

103. Huang Y, Dmochowski JP, Su Y, Datta A, Rorden C, Parra LC. Automated MRI segmentation forindividualized modeling of current flow in the human head. J Neural Eng. (2013) 10:66004-17. doi: 10.1088/1741-2560/10/6/066004

104. Datta A, Bansal V, Di J, Patel J, Reato D, Bikson M. Gyri-precise head model of transcranial direct currentstimulation: Improved spatial focality using a ring electrode versusconventional rectangular pad. Brain Stimul. (2009) 2:201-7.e1. doi: 10.1016/J.BRS.2009.03.005

105. Esmaeilpour Z, Marangolo P, Hampstead BM, Bestmann S, Galletta $\mathrm{E}$, Knotkova $\mathrm{H}$, et al. Incomplete evidence that increasing current intensity of tDCS boosts outcomes. Brain Stimul. (2018) 11:310-21. doi: 10.1016/j.brs.2017.12.002

106. Knotkova H, Riggs A, Berisha D, Borges H, Bernstein H, Patel V, et al. Automatic M1-SO montage headgear for transcranial direct current stimulation (TDCS) suitable for home and high-throughput inclinic applications. Neuromodulation. (2019) 22:904-10. doi: 10.1111/ner. 12786

Conflict of Interest: Authors AD and MB have equity in Soterix Medical Inc.

The remaining authors declare that the research was conducted in the absence of any commercial or financial relationships that could be construed as a potential conflict of interest.

Publisher's Note: All claims expressed in this article are solely those of the authors and do not necessarily represent those of their affiliated organizations, or those of the publisher, the editors and the reviewers. Any product that may be evaluated in this article, or claim that may be made by its manufacturer, is not guaranteed or endorsed by the publisher.

Copyright (C) 2022 DaSilva, Datta, Swami, Kim, Patil and Bikson. This is an openaccess article distributed under the terms of the Creative Commons Attribution License (CC BY). The use, distribution or reproduction in other forums is permitted, provided the original author(s) and the copyright owner(s) are credited and that the original publication in this journal is cited, in accordance with accepted academic practice. No use, distribution or reproduction is permitted which does not comply with these terms. 